\title{
Exploring phases of dense QCD with compact stars
}

\author{
Armen Sedrakian ${ }^{1, a}$ \\ ${ }^{1}$ Institute for Theoretical Physics, J. W. Goethe-University, D-60438 Frankfurt-Main, Germany
}

\begin{abstract}
I review a number of recent developments in the physics of compact stars containing deconfined quark matter, including (a) their cooling with possible phase transition from a fully gapped to a gapless phase of QCD at low temperatures and large isospin; (b) the transport coefficients of the $2 \mathrm{SC}$ phase and the role played by the Aharonov-Bohm interactions between flux-tubes and unpaired fermions; (c) rapidly rotating compact stars and spin-down and spin-up induced phase transition between hadronic and QCD matter as well as between different phases of QCD.
\end{abstract}

\section{Introduction}

A unique way to discern the phase structure of dense quantum chromodynamics (QCD) is offered by the phenomenology of compact stars. Their global parameters, such as the mass, the radius, the moment of inertia etc. depend sensitively on the equation of state (i.e. pressure vs density relation at zero temperature; the many-body methods to derive an equation of state appropriate for compact stars are reviewed, for example, in Refs. [1-3]). The recent measurements of pulsars masses in binary orbits with white dwarfs inferred masses in the range 1.8 - 2.0 solar masses (hereafter $M_{\odot}$ ) [4-6]. These observations suggest that the equation of state of densest regions of compact stars must be moderately stiff. There are observational indications that the pulsar radii are not very large, possibly in the range of $11 \leq R \leq 14 \mathrm{~km}$, which suggest that at lower densities the equation of state cannot be too stiff. The mass bounds quoted above were important in constraining various models of equations of state of dense matter in recent years.

The information gained from the global parameters of compact stars is however limited, as the equation of state may be insensitive to the detailed structure of QCD phases, which is dictated by the form of the quasiparticle spectrum in the vicinity of the Fermi surface. An obvious example is the pairing, which introduces a gap in the spectrum of quasiparticles and leads to the phenomenon of superconductivity of quarks [7-10]. The cooling and transport in compact stars is sensitive to such details and most of this review is dedicated to the understanding of these properties of various phases of dense QCD.

This review is structured as follows. Section 2 discusses the cooling of compact stars with quark cores, in particular the differences in the neutrino emission from phases with and without fully gapped Fermi spheres. Section 3 reviews the recent computations of the transport coefficients of two-flavor cold quark matter where two of the colors are paired (the so-called 2SC phase). The possible role of color-magnetic flux tubes is also discussed. Finally, in Sec. 4 we discuss the possibility of observing a

\footnotetext{
ae-mail: sedrakian@th.physik.uni-frankfurt.de
} 
phase transition between the hadronic and superconducting quark matter as well as phase transitions between two different phases of dense QCD in spinning up and down compact stars. Some final remarks are given in Sec. 5. An earlier account of the topics listed above can be found in Ref. [11]. This review does not provide a comprehensive coverage of the subjects listed above and is focused on the research of the author.

\section{Cooling of compact stars}

Currently the deconfinement of baryons into quark is not well understood, consequently the densities at which such a phase transition can take in compact stars is uncertain. The scale at which we may expect such a transition is set by the characteristic size of a baryon, which should be $\leq 0.84 \mathrm{fm}$ - the measured charge radius of the proton. Once the interparticle distance becomes of the order of this scale the baryons will lose their identity, as their wave-functions will start to overlap. Furthermore, the phase diagram of quark matter at not very high densities is not precisely known, because at the densities relevant to compact stars the perturbative QCD is not valid and non-perturbative methods (such as lattice QCD) face technical obstacles.

Cooling of compact stars can provide indirect information on the properties of quark phases if the density of deconfinement is indeed reached in some heavier stars. Such stars are known as hybrid compact star. In this Section we summarize the results of the studies of cooling of hybrid compact stars carried out in Refs. [12-14]. As part of this program, the rapid cooling of the compact star in Cassiopea A (hereafter Cas A) was modelled as a phase transition within the QCD phase diagram [13]. The latest updated data [15] that covers the 10 year period from 2003 to 2013 was fitted in Ref. [14]. The unprecedented fast cooling of the compact star in Cas A, in fact, requires fast transient cooling model for this object consistent with the estimate of Ref. [15], which indicates a decline in the temperature of the star $2.9 \pm 0.9 \%$ over 10 years of observation. However, this data cannot be interpreted unambiguously for a number of reasons, see the discussion in Refs. [15, 16].

Before turning to the QCD based description of cooling of compact stars and the Cas A case, it should be mentioned that a number of alternative models describe theoretically the Cas A cooling behaviour on the basis of physics unrelated to QCD degrees of freedom. Nucleonic stellar models (i.e., those containing neutrons, protons, and electrons) attribute the rapid cooling to the onset of Cooper pair-breaking process in the neutron superfluid component [17-22]. An alternative nucleonic model of Ref. [23] uses rates of modified Urca and bremsstrahlung processes that are enhanced compared to rates used in the minimalistic models quoted above by several orders of magnitude due to a softening of pionic modes, as initially discussed in Ref. [24]. A further model requires a fine-tuned onset of Urca process in rotating compact stars [25].

We consider models of hybrid compact stars which contain a color superconducting quark core surrounded by a nucleonic envelope. These phases are separated by a sharp interface, see Ref. [26]. Stars with such hybrid structure naturally correspond to the most massive members of the sequence of stellar equilibria modelled with an equation of state which contains a phase transition from nucleonic to quark matter. These massive members must be heavy enough to account for the inferred masses of pulsars [4-6].

Non-superconducting quarks cool matter via the direct Urca processes $d \rightarrow u+e+\bar{v}$ and $u+$ $e \rightarrow d+v$ at a rate which is much larger than for the ordinary nucleonic matter with low proton fraction [12, 27-30]. However, cold quark matter is a (color) superconductor [7-10]. Because of $\beta$-equilibrium and non-zero strange quark mass, the Fermi surfaces of up and down quarks are shifted by amount which is of the order of the electron chemical potential or strange quark mass. This implies that new superconducting phases will emerge, in which the Cooper pairing differs from the 


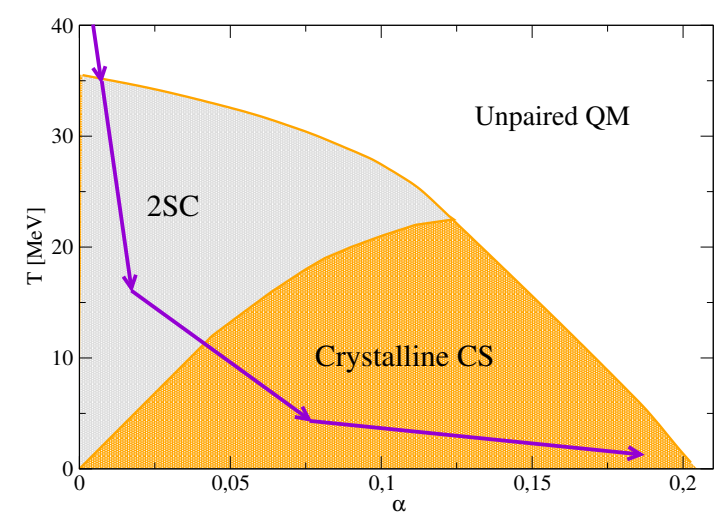

Figure 1. The 2SC and FF phases in the phase diagram of two-flavor quark matter spanned by the isospin asymmetry $\alpha=\left(n_{d}-n_{u}\right) /\left(n_{u}+n_{d}\right)$, where $n_{d}$ and $n_{u}$ are the number densities of $d$ and $u$ flavors of quarks, and temperature. The path that a compact star traverses during the cooling process is shown by arrows.

ordinary Bardeen-Cooper-Schrieffer pairing. Examples are the gapless two-flavor phases [31, 32] or the the crystalline color-superconducting phase $[33,34]$. The latter phase has a number of realizations, because there are many ways to break the spatial symmetries by allowing Cooper pairs to carrying finite momentum. Ref. [14] assumed the so-called Fulde-Ferrell phase (hereafter FF phase), which postulates a single plane wave modulation of the condensate. As far as the cooling is concerned, the general properties of crystalline phases - the existence of gapless excitations [33-36] - is present already in the FF model.

A compact star born in a supernova explosion is hot and matter is nearly isospin symmetrical. In the subsequent minutes to hours the matter cools rapidly and the isospin asymmetry increases to the values characteristic for neutron stars. For temperatures $T \leq T_{c}$, where $T_{c}$ is the critical temperature of phase transition, and not too high isospin asymmetries one finds the homogeneous 2SC phase in which the rotational/translational symmetries are unbroken, see Fig. 1. For low temperature and large asymmetries one finds that the ground state corresponds to the FF state.

The consequences of the transition from the symmetrical two-flavor BCS phase to the crystalline phase at some temperature $T^{*}$ for the cooling of hybrid stars with application to the Cas A case were studied in Ref. [14]. Fits were performed to obtain the value of this temperature. Because the crossflavor pairing occurs between the green and red colored quarks and the blue quarks do not participate in this type of the pairing a second parameter - the gap for blue-colored quarks $\Delta_{b}$ - was introduced in the color $\mathbf{6}_{S}$ and flavor $\mathbf{3}_{S}$ channel. Note that this same-flavor and same-color pairing is unaffected by the flavor asymmetry [37]. An addition parameter $w$ is introduced to account for the finite time-scale of the phase transition, see Ref. [14] for details. Illustrative results are shown in Fig. 2, where we display the dependence of the (redshifted) surface temperature on time for a stellar model with mass $M / M_{\odot}=1.93$. The parameter $T^{*}$ is varied as indicated in the figure whereas the parameters $w$ and $\Delta_{b}$ are held fixed.

For small $T^{*}$ the quark core has no influence on the cooling because the neutrino emission from the red-green and blue condensates is suppressed by the pairing gaps in these channels. In the opposite case of high values of $T^{*} \sim 0.2 \mathrm{MeV}$ the transition to the FF phase occurs early in time and fast neutrino emission via the direct Urca process cools the star rapidly below the value measured for Cas A. After fine tuning of the phase-transition temperature to the value $T^{*}=0.009385 \mathrm{MeV}$ the temperature of Cas A can be reproduced accurately (Fig. 2 left panel). As seen from the right panel of Fig. 2 a further tuning of the parameter $T^{*}$ allows us to fit the details of the data with good accuracy.

Thus, we conclude that the rapid cooling of the compact star in Cas A can be accounted for via the phase transition from the $2 \mathrm{SC}$ to FF-type phase. It is interesting that the phase transition takes place within the phase diagram of $Q C D$ and is the consequence of the ordering of various 

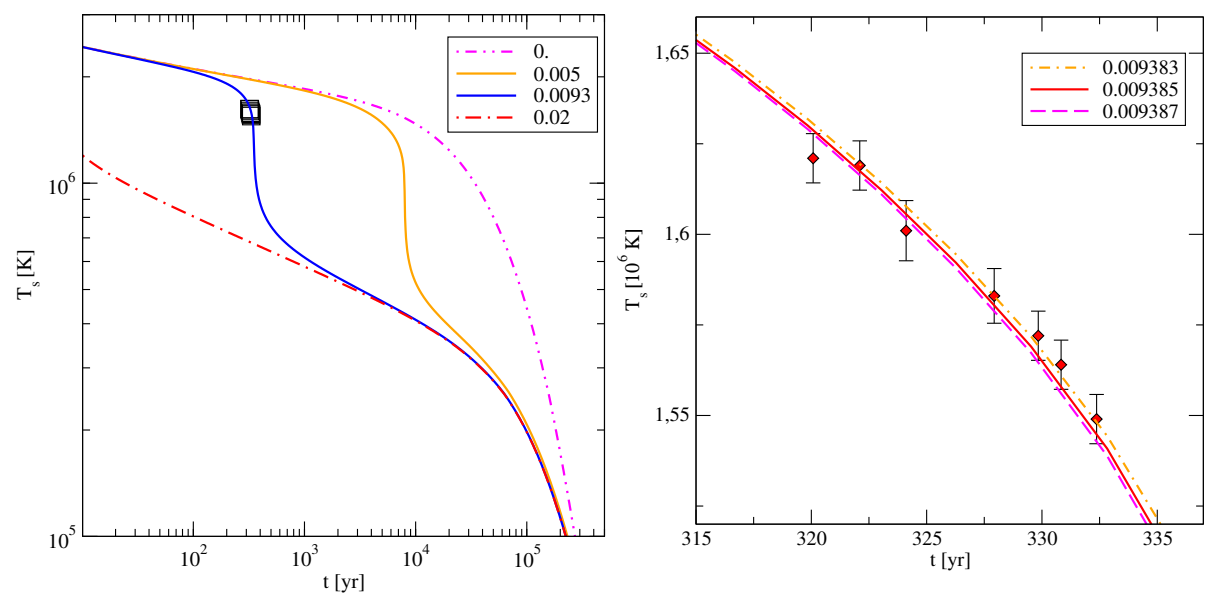

Figure 2. Dependence of the surface temperature of a CSs given in $\mathrm{K}$ on time in year. The lines correspond to different phase transition temperature $T^{*}$ for fixed values of the width $w=0.2$ and the blue quark pairing gap $\Delta_{b}=0.15$ (both in $\mathrm{MeV}$ ). The labels correspond to values of $T^{*}$ in $\mathrm{MeV}$. The Cas A data is shown by squares.

superconducting phases in the temperature, density, and isospin spaces. Such ordering was observed in numerous studies of other population imbalance systems, such as the nucleonic superfluids under isospin asymmetry or strong magnetic fields [38, 39].

In addition to the study of Cas A behaviour, Ref. [14] studied the dependence of the cooling curves on the variations of the mass of the compact star model. It was found that the heavy stars that are close to the maximal mass will cool to lower temperatures much faster; therefore some refitting of the parameters will be required to adjust these models to the case of Cas A. It was also found that low mass stars $M \sim 1.1 M_{\odot}$ remain warm over longer time scales and are thus hotter than their heavy counterparts.

Some issues remain to be studied, for example the role of strange quarks in the quark phases. These may influence the cooling in case they are not involved in the pairing pattern. However, at asymptotically high density, when the strange quark mass vanishes, the color-flavor-locked (CFL) phase in which the pairing is "perfect", in the sense that all colors and flavors are involved in the condensate, is the ground state of matter [40]. Because the CFL phase has very low heat capacity as well as neutrino emissivity strong modifications are expected only in the case where imperfect CFL phases arise, such as the gapless CFL phase [10]. In any event, the low number of strange quarks can render the process on strange quarks subdominant.

\section{Transport phenomena}

The treatment of transport phenomena in dense QCD phases of neutron stars requires the knowledge of excitation spectra of quarks near their Fermi-surface. The pairing patterns in the color and flavor spaces are essential in determining the content of excitations, especially those that are ungapped. At large densities the dominant CFL phase is a superfluid, whose excitation spectrum contains only phonons in analogy liquid ${ }^{4} \mathrm{He}$, see Refs. [41-43]. As the density is lowered to the densities relevant to compact stars the precise from of pairing becomes uncertain. The most robust phase at this density is the the two-flavor color-superconducting (2SC) phase, which pairs up and down quarks in a color 
antitriplet state. The blue color is either unpaired or undergoes single-flavor and color pairing with very small gaps of the order of $\mathrm{keV}$ [7]. In addition to the bulk fermions, the 2SC phase can contain color-magnetic flux tubes which will carry partially the magnetic field through the color superconductor [44]. The flux tubes can effectively scatter bulk fermions and, therefore, contribute to the transport and relaxation in a certain parameter range, which we will discuss below.

\subsection{Bulk fermions}

The bulk transport coefficients of the 2SC phase were computed in Ref. [45] using the Boltzmann equation in the transport relaxation approximation. The matrix elements of the scattering processes were computed to the leading order in the electromagnetic and strong coupling $\left(\alpha\right.$ and $\alpha_{s}$ ). The screening of these interactions were taken in the Hard-Thermal-Loop approximation. In addition it was assumed that the matter is strongly degenerate with temperature $T \ll \mu_{q}$, where $\mu_{q}$ is the chemical potential of quarks. An important feature of the interactions is the mixing of the gauge fields into a linear combination of electromagnetism and gluonic fields, called $\tilde{Q}$ and $X$. The first field is unscreened in the 2SC phase and penetrates unimpeded in the bulk; the second field is Meissner screened over the distances of the order of the penetration depth.

The ungapped fermions of the 2SC phase are the blue up $(u)$ and down $(d)$ quarks and electrons. Ref. [45] found the following key features of the transport in the 2SC phase: the thermal conductivity and shear viscosity are dominated by the blue- $d$ quarks for sufficiently low temperatures. At higher temperatures the electrons take over the dominant role. This transition for thermal conductivity occurs at $T / \mu_{q} \sim \alpha / 7.7 \sim 10^{-3}$, so most of the temperature range of interest for neutron stars is in the blue- $d$ dominated regime with the scaling $\kappa_{b d}=0.00617 /\left(T / \mu_{q}\right)$. For the shear viscosity the crossover from blue- $d$ to electron domination occurs at $T / \mu_{q} \sim 10^{-5}$, so electrons become dominant at relatively low temperature $T \sim 10 \mathrm{keV}$; the shear viscosity scales as $\eta_{e} \simeq 0.00231\left(\mu_{q}^{4} / 5 \pi^{2}\right) \alpha^{-5 / 3} T^{-1}\left(T / \mu_{q}\right)^{-2}$. On the other hand the electrical conductivity of the rotated $\tilde{Q}$ charge is completely dominated by the electron, because the blue- $d$ quarks are $\tilde{Q}$ neutral, whereas blue- $u$ quarks are much less abundant; the electrical conductivity of electrons is given by $\sigma_{e}=0.0433\left(\mu_{q}^{2} e^{2} / \pi^{2}\right) \alpha^{-5 / 3} T^{-1}\left(T / \mu_{q}\right)^{-2 / 3}$; see Ref. [45] for the expressions of less dominant contributions. It was also shown that $S U(2)_{r g}$ gluons do not contribute to the transport coefficients in 2SC quark matter significantly.

\subsection{Color-magnetic flux-tubes}

The magnetic field will penetrate the $2 \mathrm{SC}$ phase in form of quantized color-magnetic flux tubes because for realistic values of parameters the $2 \mathrm{SC}$ phase is a type-II $X$-charge superconductor [44]. The corresponding Ginzburg-Landau parameter is given by

$$
\kappa_{2 \mathrm{SC}} \approx 11 \frac{\Delta}{\mu_{q}} \geq \frac{1}{\sqrt{2}} .
$$

where $\Delta$ is the gap in the red-green channel. The critical field for formation of flux tubes is very high, $H_{c 1} \sim 10^{17} \mathrm{G}$ compared to typical field of a neutron stars, but as the 2SC phase nucleates, domains of superconducting matter will form, which will be Meissner screened. This will leave the normal regions with intense magnetic fields above the average value. Once these regions eventually undergo superconducting phase transition the magnetic field intensity within them will be larger than $H_{c 1}$ with $X$ flux trapped in the form of flux tubes. The detailes of the configurations and density of the $X$-tubes is not known and depends, among several things, on the dynamics of the phase transition. However, their average density is expected to be of the order of that of the ordinary flux tubes in the hadronic core by simple flux conservation. 


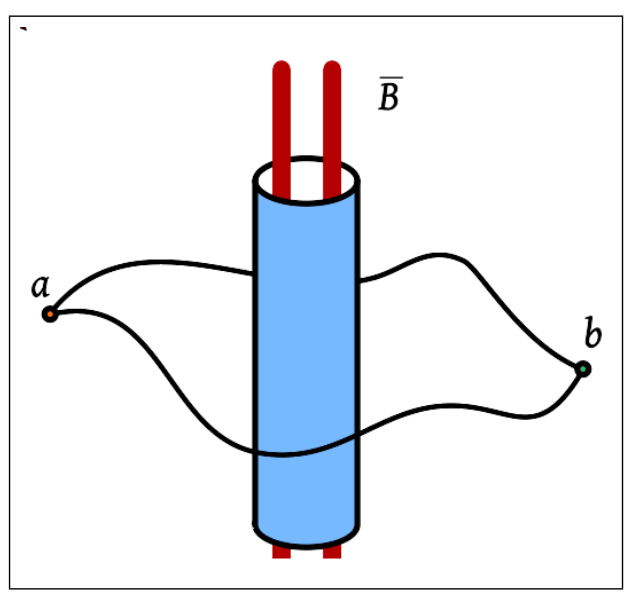

Figure 3. Illustration of the Aharonov-Bohm effect: an electron or blue $u$-quark acquires a phase shift as it passes from point $a$ to point $b$ around a color-magnetic flux tube (shown as the cylindrical slab).

The Aharonov-Bohm scattering cross-section of gapless fermions with the $X$ flux tubes was calculated in terms of the cross-section given by

$$
\frac{d \sigma}{d \vartheta}=\frac{\sin ^{2}(\pi \tilde{\beta})}{2 \pi k \sin ^{2}(\vartheta / 2)},
$$

where $k$ is the momentum in the plane perpendicular to the flux-tube, $\vartheta$ is the scattering angle, $\tilde{\beta}=$ $q_{p} / q_{c}, q_{p}$ is the charge of the scattering particle and $q_{c}$ is the charge of the condensate whose winding by a phase of $2 \pi$ characterizes the flux-tube. The values of $\tilde{\beta}$ are given by

$$
\tilde{\beta}=\operatorname{diag}\left(\frac{1}{2}+\frac{3}{2} \sin ^{2} \varphi, \frac{1}{2}-\frac{3}{2} \sin ^{2} \varphi, \frac{1}{2}-\frac{3}{2} \sin ^{2} \varphi, \frac{1}{2}+\frac{3}{2} \sin ^{2} \varphi,-1+3 \sin ^{2} \varphi,-1,-3 \sin ^{2} \varphi\right)
$$

in the basis $\psi=\left(r u, g d, r d, g u, b u, b d, e^{-}\right)$, where $r g b$ refer to red, green, and blue colors, $e^{-}$refers to electron, and the mixing angle is defined as $\cos \phi=\sqrt{3} g / \sqrt{e^{2}+3 g^{2}}$, where $e$ and $g$ are the electromagnetic and strong coupling constants.

The relaxation rate for particles of species $i$ scattering off flux tubes of area density $n_{v}$ is then given by [44]

$$
\tau_{i f}^{-1}=\frac{n_{v}}{p_{F i}} \sin ^{2}\left(\pi \tilde{\beta}_{i}\right) .
$$

where $n_{v}$ is the number density of color-magnetic flux tubes, $p_{F i}$ is the Fermi momentum of fermion $i$. This result corresponds to the standard form for relaxation rate known for classical gases $\tau^{-1}=c n_{v} \sigma$, where $c=1$ is the speed of the particles, $n_{v}$ is the density of scattering centers, and $\sigma \propto \sin ^{2}(\pi \tilde{\beta}) / p_{F}$ is the cross section of Aharonov-Bohm scattering. Eq. (4) sets the scale for the thermal relaxation of the blue quarks and electrons in the $2 \mathrm{SC}$ phase. Note however that the blue- $d$ quark, has zero AharonovBohm interaction with the flux-tubes because $\tilde{\beta}=0$. The other two, the electron and blue- $u$ quark, have the same Aharanov-Bohm factors, see Eq. (3), but different Fermi momenta.

Ref. [44] argued that because the magnetic field in hadronic envelope is below the lower critical field $H_{c 1}$ for $X$-flux-tubes into 2SC quark matter, the trapped flux-tubes will be pulled out of the quark core. The force pulling the flux-tubes is balanced by the mutual friction force on the moving flux-tube due to its Aharonov-Bohm interaction with electrons and blue- $u$ quarks. Apart from the rearrangement of the magnetic field, which is characterized by time-scales comparable to pulsar life-times, the flux expulsion has other physical significance: it could be a significant source of heating of the lower base 
of the nuclear mantle, and therefore the inner core (which is roughly isothermal after $10^{3} \mathrm{yr}$ ). Internal heating is known to affect the thermal evolution of compact star in the later stages of their thermal evolution (photo cooling era).

Consider now the combined effect of scattering of a fermion off other fermions and flux-tubes. If there are several scattering channels then the net relaxation time for fermion species $i$ is given by the

$$
\tau_{i}^{-1}=\tau_{i, v}^{-1}+\sum_{j} \tau_{i j}^{-1}
$$

where $\tau_{i, v}^{-1}$ and $\tau_{i j}^{-1}$ are the fermion-flux-tubes and fermion-fermion relaxation rates, respectively. In a first approximation one can assume that the fermion-fermion and fermion-flux-tubes scattering do not interfere. Then, it is clear that the fermion-flux-tubes scattering shortens the mean-free-path of the partciles and, therefore, suppresses the transport coefficients.

With the picture above, some conclusions can be drawn for the individual transport coefficients [45]. Because the dominant contribution to the thermal conductivity is from blue- $d$ quarks, which have zero Aharanov-Bohm scattering with vortices, the scattering off the flux-tubes has no significant effect on the thermal conductivity. The electrical conductivity and shear viscosity are dominated by electrons where the interactions are dominated by the $\tilde{Q}$ interaction.

The electron-flux-tubes relaxation rates for electrical conductivity and shear viscosity are approximately the same, as these involve the momentum relaxation up to a constant of order 1. Clearly the electron-flux-tubes sattering becomes important, when its relaxation time is comparable to the fermion-fermion relaxation time. According to the estimates of Ref. [45] flux-tubes can lower the magnitude of transport coefficients for realistic values of the external magnetic field.

\section{Spin-up and spin-down induced phase transitions}

The studies of compact stars with quark cores in a wide range of rotation periods from the Keplerian limit down to the static limit is of great interest in the interpretation of the phenomena related to the physics of pulsars. Compact stars that are born rapidly rotating evolve adiabatically by losing energy to gravitational waves and electromagnetic (to lowest order, dipole) radiation. Such evolution can be approximated by a sequence of configurations with constant rest mass and varying spin. The massive rapidly rotating configurations may not have a stable static limit. Consequently, the spindown evolution can lead to the formation of a black hole when the support through centrifugal force cannot balance the pull of gravity anymore. The configurations which do not have static counterparts are known as supramassive configurations [46]. The time-reversed counterpart of the spin-down scenario is also realized in nature. According to the formation scenario of the millisecond pulsars, these are spun-up to millisecond frequencies by accretion from a companion. In this case the evolution involves some mass transfer via accretion to the compact star, therefore the rest mass of the star is not a constant anymore. However, the changes in the rest mass during the accretion are not significant and do not change the qualitative picture. Therefore, the changes in the structure of the compact stars as the star spin increases with time can be considered, in a first approximation, as "time reversed" evolution, which does not require additional physical input, such as the mass accretion rate from the companion.

How the spin evolution of hybrid stars, i. e., stars with quark-matter cores surrounded by a nuclear envelope, may differ from the evolution of purely nucleonic stars? The most dramatic effect is the spin-down or spin-up induced phase transition(s) between the hadronic and quark matter phases. The reason is that rapidly rotating stars are less compressed and are effectively more "low-density" objects than their static counterparts. Changes in the spin of the star induce changes in its density profile, 


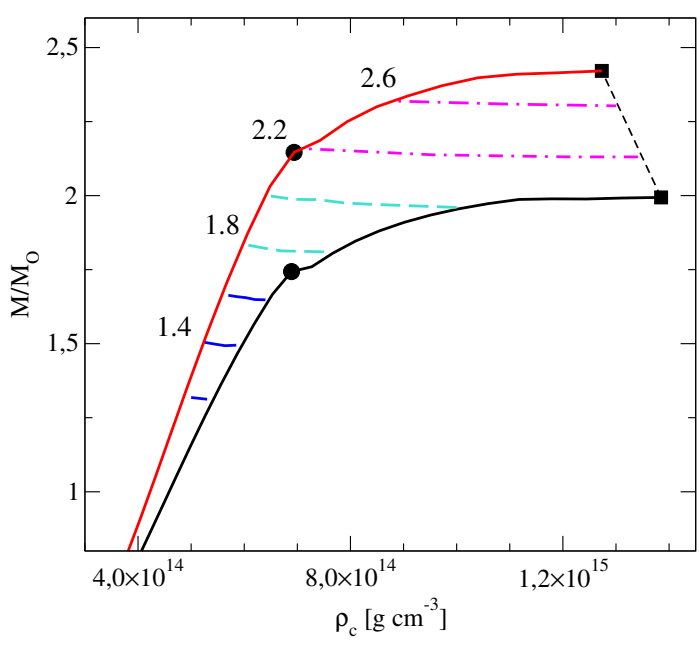

Figure 4. Dependence of masses of hybrid compact stars on the central density. The lower and upper solid lines corresponds to non-rotating and maximally rotating stars. The dashed and dash-dotted lines show stars with a fixed rest masses, as labeled in the plot, with the mass step between two sequential lines equal 0.2 . Among these the dashed-dotted lines correspond to the class of the stars which do not have static counterparts. The squares show the maximal mass stars, which are connected by the line at which the stability region terminates. The stars located to the right of dots are hybrid stars that contain quark matter. All mass units are given in units of the solar mass $M_{\odot}$.

which in turn can lead to density-induced phase transitions from quark to hadronic matter and back. The physics of spin-up and -down induced phase transitions in non-superconducting quark matter was first discussed discussed by Glendenning, Pei and Weber in Ref. [47] and subsequently elaborated in many studies, see e. g., Refs. [48-52].

Rotating compact stars containing color superconducting cores were considered initially in Ref. [53]. The color superconducting matter was taken to be in the crystalline phase. The case where the quark matter pairs either in the $2 \mathrm{SC}$ or the CFL phases was subsequently studied in Ref. [54] on the basis of an equation of state constructed to reproduce the nuclear and quark matter phenomenologies as well as the astrophysical constraint on the value of the maximum mass of a compact star $M / M_{\odot} \geq 2$. Below, our discussion follows the study of Ref. [54], where the equation of state of a hybrid star was constructed assuming a sharp interface between the phases. This is the case when the surface tension of the interface is not too small. The hadronic matter description is based on relativistic density functional of nuclear matter with density-dependent couplings, see Refs. [55, 56] for the parameterization and further details. The quark matter equation of state is based on the Nambu-JonaLasinio model which includes in addition to common interactions also repulsive vector interactions as given in Ref. [26]. The mass vs central density relations for hybrid stars are shown in Fig. 4 in the static and Keplerian limits. These limits can be connected by constant rest mass lines (near horizontal lines in Fig. 4), which correspond to the scenarios of spin-up or spin-down of the stars without mass transfer. There are three classes of constant rest mass sequences in the case of hybrid stars:

1. The well-known supramassive compact stars which are stable only due to the (uniform) rotation (dashed-dotted lines in Fig. 4) . They collapse to a black hole as they spin down below certain critical angular velocity; the instability sets-in in the region to the right of the line connecting the maximal masses of non-rotating and maximally fast rotating compact stars in Fig. 4.

2. The second class can be defined as transitional compact stars (dashed lines in Fig. 4). These are purely nucleonic in the limit of maximally fast rotation and are hybrid in the static limit. This implies that there is a phase transition from nucleonic phase to the quark matter phase at the center of the star as the star spins down or up. Note that phase transition take also place within the supramassive sequences which contain quark matter already in the limit of maximal rotation. Because the central density and the density profiles of these stars change with their 


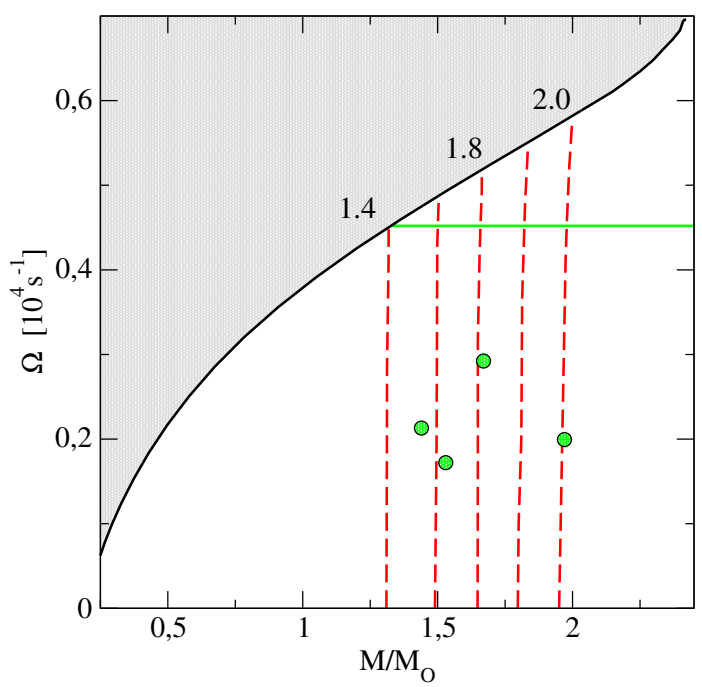

Figure 5. Frequency vs mass diagram for compact stars. Only the most massive object J1614-2230 is massive enough to contain quark matter. The solid line indicates the Keplerian frequency as a function of mass; the area above the line is excluded for uniformly rotating stars.

spin there is a continuous conversion of quark matter to nucleonic matter and back depending on whether the star spins down or up.

3. The third class of stars do not contain quark matter in either limit, i.e., these are purely nucleonic as the central densities are not sufficiently large for quark matter to form (solid lines in Fig. 4). The masses of these stars are below $1.7 M_{\odot}$ for the equation of state used in our example.

Figure 5 displays the frequency vs mass diagram for compact stars, with dots corresponding to objects for which the mass and the spin frequency are measured, see Ref. [54] for details. The shaded region is inaccessible to our models in the case of uniform rotation. The horizontal line shows the spin frequency of the fastest rotating pulsar J1748-2446, for which the mass is unknown. For present equation
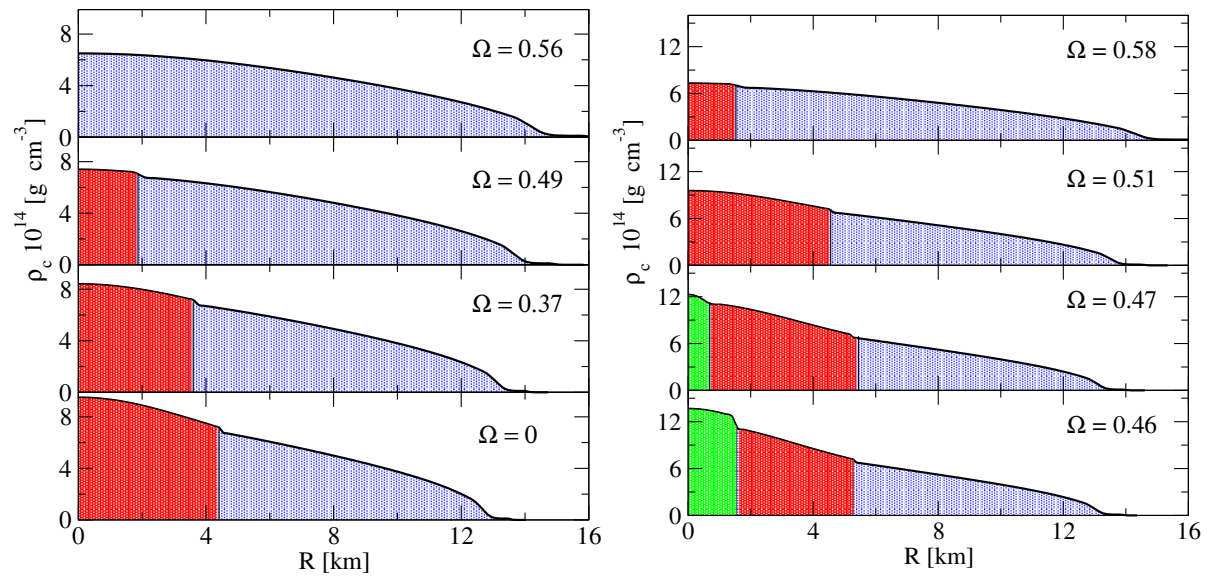

Figure 6. Equatorial density profiles of a rotating compact stars with rest masses $2.2 M_{\odot}$ (left panel) and 2.4 $M_{\odot}$ (right panel). The low-density region corresponds to nucleonic matter (blue), which is followed by the 2SC phase (red) and CFL phase (green). The frequencies are given in units of $10^{4} \mathrm{~s}^{-1}$. 
of state this object's mass needs to be above $1.4 M_{\odot}$, which illustrates the possibility of constraining the equations of state of dense matter by future measurements of the spin of sub-millisecond pulsars.

Figure 6 shows the dependence of the equatorial density profile of $2.2 M_{\odot}$ (left panel) and 2.4 $M_{\odot}$ (right panel) rest-mass stars on the internal radius for four spin frequencies in the range $0 \leq \Omega \leq$ $5.6 \times 10^{3} \mathrm{~Hz}$. The upper panels in both cases show the stars rotating at the Keplerian limit. As expected, the region occupied by the $2 \mathrm{SC}$ phase becomes larger as the star's spin is reduced. Eventually, the CFL phase may form at the center of the most massive stars. Because the phase transition from nucleonic phase to the 2SC phase and from the 2SC to the CFL phase is first order, both are accompanied by jumps in the density, as seen in Fig. 6

\section{Final remarks}

The exploration of the dense QCD via the astrophysics of compact stars is an actively pursued subject. We expect substantial observational progress in the studies of compact stars in the upcoming years, including further measurements of the masses of pulsars, the radii of neutron stars, and eventually the gravitational wave signals from the mergers of two neutron stars or neutron stars and black holes. This motivates strongly further studies of dense matter in all its facets, including the physics that goes beyond the equation of state. Better understanding of the transport coefficients of dense QCD, weak interactions in dense matter, and dynamics of phase transitions would largely improve our modelling of astrophysical phenomena that are relevant for the current observational programs.

\section{Acknowledgments}

I would like to thank my collaborators M. Alford, L. Bonanno, G. Colucci, X.-G. Huang, H. Nishimura, and D. Rischke, for their contribution and insight into the research described above. This work was supported by the Deutsche Forschungsgemeinschaft (Grant No. SE 1836/3-2), by the Helmholtz International Center for FAIR, and by the NewCompStar COST Action MP1304.

\section{References}

[1] F. Weber, Pulsars as astrophysical laboratories for nuclear and particle physics, IOP, Bristol (1999).

[2] A. Sedrakian, Prog. Part. Nucl. Phys. 58, 168 (2007).

[3] J. M. Lattimer and M. Prakash, Phys. Rep. 621, 127 (2016).

[4] P.B. Demorest, T. Pennucci, S.M. Ransom, et al., Nature 467, 1081 (2010).

[5] J. Antoniadis, P.C.C. Freire, N. Wex, et al., Science 340, 448 (2013).

[6] E.D. Barr, P.C.C. Freire, M. Kramer, et al., arXiv: 1611.03658 (2016).

[7] D. Bailin, A. Love, Phys. Rep. 107, 325 (1984).

[8] K. Rajagopal, F. Wilczek, arXiv:hep-ph/0011333.

[9] M. Alford, Annual Rev. of Nucl. and Part. Science 51, 131 (2001).

[10] M.G. Alford, A. Schmitt, K. Rajagopal, T. Schäfer, Rev. Mod. Phys. 80, 1455 (2008).

[11] A. Sedrakian, PoS (Confinment X), 251 (2013), arXiv: 1301.2675.

[12] D. Hess, A. Sedrakian, Phys. Rev. D 84, 063015 (2011).

[13] A. Sedrakian, A\&A 555, L10 (2013).

[14] A. Sedrakian, Eur. Phys. J. A 52, 44 (2016).

[15] K.G. Elshamouty, C.O. Heinke, G.R. Sivakoff, et al., ApJ 777, 22 (2013). 
[16] B. Posselt, G.G. Pavlov, V. . Suleimanov, O. Kargaltsev, ApJ 779, 186 (2013).

[17] D. Page, M. Prakash, J.M. Lattimer, A.W. Steiner, Phys. Rev. Lett. 106, 081101 (2011).

[18] P.S. Shternin, D.G. Yakovlev, C.O. Heinke, et al., MNRAS 412, L108 (2011).

[19] P.S. Shternin, D.G. Yakovlev, MNRAS 446, 3621 (2015).

[20] W.C.G. Ho, K.G. Elshamouty, C.O. Heinke, A.Y. Potekhin, Phys. Rev. C 91, 015806 (2015).

[21] W.G. Newton, K. Murphy, J. Hooker, B.A. Li, ApJ Lett. 779, L4 (2013).

[22] L.B. Leinson, Phys. Lett. B 741, 87 (2015).

[23] D. Blaschke, H. Grigorian, D.N. Voskresensky, F. Weber, Phys. Rev. C 85, 022802 (2012).

[24] C. Schaab, D. Voskresensky, A.D. Sedrakian, F. Weber, M.K. Weigel, A\&A 321, 591 (1997).

[25] R. Negreiros, S. Schramm, F. Weber, Phys. Lett. B 718, 1176 (2013).

[26] L. Bonanno, A. Sedrakian, A\&A 539, A16 (2012).

[27] D. Page, M. Prakash, J.M. Lattimer, A.W. Steiner, Phys. Rev. Lett. 85, 2048 (2000).

[28] M. Alford, P. Jotwani, C. Kouvaris, J. Kundu, K. Rajagopal, Phys. Rev. D 71, 114011 (2005).

[29] R. Anglani, G. Nardulli, M. Ruggieri, M. Mannarelli, Phys. Rev. D 74, 074005 (2006).

[30] H. Grigorian, D. Blaschke, D. Voskresensky, Phys. Rev. C 71, 045801 (2005).

[31] I. Shovkovy, M. Huang, Phys. Lett. B 564, 205 (2003).

[32] H. Müther, A. Sedrakian, Phys. Rev. D 67, 085024 (2003).

[33] M. Alford, J.A. Bowers, K. Rajagopal, Phys. Rev. D 63, 074016 (2001).

[34] R. Anglani, R. Casalbuoni, M. Ciminale, et al., Rev. Mod. Phys. 86, 509 (2014).

[35] A. Sedrakian, D.H. Rischke, Phys. Rev. D 80, 074022 (2009).

[36] X.G. Huang, A. Sedrakian, Phys. Rev. D 82, 045029 (2010).

[37] M.G. Alford, J.A. Bowers, J.M. Cheyne, G.A. Cowan, Phys. Rev. D 67, 054018 (2003).

[38] M. Stein, A. Sedrakian, X.G. Huang, J.W. Clark, Phys. Rev. C 93, 015802 (2016).

[39] M. Stein, A. Sedrakian, X.G. Huang, J.W. Clark, Phys. Rev. C 90, 065804 (2014).

[40] M. Alford, K. Rajagopal, F. Wilczek, Nucl. Phys. B 537, 443 (1999).

[41] I.A. Shovkovy, P.J. Ellis, Phys. Rev. C 66, 015802 (2002).

[42] C. Manuel, F.J. Llanes-Estrada, J. Cosmo. Astropart. Phys. 8, 001 (2007).

[43] C. Manuel, A. Dobado, F.J. Llanes-Estrada, JHEP 9, 076 (2005).

[44] M.G. Alford, A. Sedrakian, J. of Phys. G 37, 075202 (2010).

[45] M.G. Alford, H. Nishimura, A. Sedrakian, Phys. Rev. C 90, 055205 (2014).

[46] G.B. Cook, S.L. Shapiro, S.A. Teukolsky, ApJ 422, 227 (1994).

[47] N.K. Glendenning, S. Pei, F. Weber, Phys. Rev. Lett. 79, 1603 (1997).

[48] N.K. Glendenning, F. Weber, ApJ Lett. 559, L119 (2001).

[49] H. Grigorian, D. Blaschke, G. Poghosyan, Nucl. Phys. A 715, 831 (2003).

[50] J.L. Zdunik, M. Bejger, P. Haensel, E. Gourgoulhon, A\&A 450, 747 (2006).

[51] E.B. Abdikamalov, H. Dimmelmeier, L. Rezzolla, J.C. Miller, MNRAS 392, 52 (2009).

[52] F. Weber, M. Orsaria, R. Negreiros, ArXiv e-prints (2013), 1307.1103.

[53] N.D. Ippolito, M. Ruggieri, D.H. Rischke, et al., Phys. Rev. D 77, 023004 (2008).

[54] N.S. Ayvazyan, G. Colucci, D.H. Rischke, A. Sedrakian, A\&A 559, A118 (2013).

[55] G.A. Lalazissis, T. Nikšić, D. Vretenar, P. Ring, Phys. Rev. C 71, 024312 (2005).

[56] G. Colucci, A. Sedrakian, Phys. Rev. C 87, 055806 (2013). 